\title{
The Role of Positron Emission Tomography/Computed Tomography (PET/CT) in Assessment of Therapeutic Response in Non-Hodgkin Lymphoma
}

\author{
HUSSEIN A. FADHIL, M.Sc.; MOHAMMED A. ALI, M.D. and AHMED M. BASSIOUNY, M.D. \\ The Department of Radiology, Faculty of Medicine, Ain Shams University, Cairo, Egypt
}

\begin{abstract}
Background: Lymphoma is the most common primary hematopoietic malignancy, make up a heterogeneous group of neoplastic diseases of lymphocytes origin broadly divided into Hodgkin Disease (HD) and Non-Hodgkin's Lymphoma (NHL) In the past decade, functional imaging with 18F-FDG PET has been the fastest growing diagnostic modality in oncology, the addition of PET to CT adds sensitivity and specificity in tumor imaging. Thus, PET/CT is a more accurate test than either of its individual components.
\end{abstract}

Aim of Study: This study was undertaken to evaluate the role the PET/CT in assessment of response to chemotherapy and or radio therapy, follow-up and assessment of remissions and relapses in patients with lymphoma.

Patients and Methods: Retrospective diagnostic interventional study in Ain Shams University Hospitals. Study population: Non Hodgkin lymphoma patients receiving therapypresenting to Ain Shams University Hospitals since Jan. 2018Apr. 2019. Patients included will perform one or more of the following: Initial PET/CT for staging and follow-up PET/CT exams. All exams will be done on the PET/CT scanner. intravenous glucose Fluorodeoxyglucose (FDG) dose depending on the protocol of department. After 60min of 18F-FDG injection MCDT diagnostic nonionic contrast examination for anatomical localization followed by PET images. The whole study took approximately 20-30 minutes.

Results: This study included 20 patients with pathologically confirmed non Hodgkin lymphoma (diffuse large B cell) who underwent PET/CT study after they had finished their treatment protocol. The PET/CT exam of each patient was interpreted for the assessment of treatment response after end of treatment. About $65 \%$ of patients received 6 cycles of chemotherapy, however only $35 \%$ received 8 cycles of chemotherapy.

In our study, 12 patients $(60 \%)$ had extra-nodal involvement and were as follow: 7 patients (55\%) had only one extra nodal affection site and 5 patients $(45 \%)$ had more than one extranodal affection site. It had high sensitivity (100\%) and specificity $(75 \%)$ for the evaluation of therapeutic response

Correspondence to: Dr. Hussein A. Fadhil, E-Mail: hussainfadhil86@gmail.com than contrast enhanced CT and fair agreement (Kappa 0.610) according to the Deauville criteria. We assess therapeutic response in our study using IHP and correlated with visual assessment using (Deauville Criteria-five-point scale).

Conclusion: Combined PET/CT using 18F-FDG is the best oncologic imaging modality at present time with indispensable role and valuable application in management of lymphoma. According to the Deauville criteria in end of treatment response assessment. We recommend in reporting of PET-CT for response assessment using the Deauville criteria.

Key Words: PET/CT - Nodal - Extranodal - LymphomaDeauville criteria - IHP (International Harmonizing Project).

\section{Introduction}

LYMPHOMA make up a heterogeneous group of neoplastic diseases of lymphocytes origin. These malignant lymphoproliferative processes are clonal B-cell, T-cell or Natural Killer (NK) cells tumors in different stages of differentiation. Lymphomas represent $6 \%$ of all neoplasms and are responsible for $3 \%$ of the mortality by oncological processes. The incidence of lymphomas is up to $4 \%$ each year, with around 6000 new cases being diagnosed annually. Lymphomas are somewhat more frequent in men than in women and more common in 2 age groups: From 15 to 40 years of age (being more frequent between 25 and 30 years) and after the age of 55 years. There are important differences among the principal types of lymphoma [1]

Lymphoma is generally divided into two groups: Hodgkin's Disease (HD) and an inhomogeneous group of conditions called Non-Hodgkin's Lymphoma (NHL). HD tends to involve a single nodal group and spread in a fixed pattern along the lymphatic chain, with infrequent extra lymph node involvement. NHL is a multifocal disease which 
often presents late with disseminated extranodal spread [2].

The Ann Arbor classification is used most often for staging. Clinical staging involves assessment of disease extent by clinical examination, history, and imaging techniques. When staging laparotomies are used as part of staging, the disease extent is designated as pathologic staging; Cotswold's modification is currently used [3].

In the past two decades, (CT) has been the main diagnostic tool in initial staging of disease and therapy follow-up in patients with cancer. Morphologic changes depicted at CT have been equated to disease manifestations. CT has the ability to depict abnormal anatomy and abnormal contrast enhancement due to pathologic changes. However, CT has limitations in depiction of pathologic changes in normal-sized structures, such as lymph nodes, and of a lesion that does not have good contrast with the surrounding tissues, which results in a reduced sensitivity of lesion detection. However, in the last decade, imaging of tumor metabolism with 2-[fluorine-18]fluoro-2-deoxy-d-glucose (FDG) Positron Emission Tomography (PET) has facilitated the identification of affected nodal and extranodal sites, even when CT has demonstrated no lesions. It also plays a role for more correct staging prior to treatment and post treatment followup [4].

The use of sensitive and specific imaging techniques for accurate initial staging and evaluation of response to therapy in patients with lymphoma is essential for their optimal management. Fluorine 18 (FDG) Positron Emission Tomography (PET) integrated with Computed Tomography (CT) has emerged as a powerful imaging tool and is being routinely used in staging, response evaluation [5]

One of the most evident advantages of FDGPET was its ability to detect significant changes in glucose metabolism or even complete remission. This enables clinicians to detect much earlier the effectiveness of a given anti-neoplastic treatment, as compared to the traditional CT imaging alone [6].

One of the most challenging aspects in the imaging of lymphomas is the assessment of response to treatment. Differentiation of tumor from fibrosis within residual radiographic masses represents a problem of interpretation for non-Hodgkin's lymphoma. Thus accurate staging is the basis for the selection of an appropriate therapeutic approach, in order to prevent over or under treatment as well as to minimize morbidity related to the radiochemotherapy regimens given [7].

Quality of life during and after treatment may also be improved when therapy is tailored appropriately. So that Positron Emission Tomography (PET) scan with the glucose analogue 2-(F-18)fluoro-2-deoxy-D-glucose (18F-FDG) has emerged as a clinical method for staging and monitoring responses to treatment [4].

Positron Emission Tomography (PET) using Fluorodeoxyglucose (FDG) with Computed Tomography (CT) has proved effective as a metabolic imaging tool with compelling evidence supporting its superiority over conventional modalities, particularly in staging and early evaluation of response to treatment $[8]$.

\section{Aim of the work:}

This study was undertaken to evaluate the role the PET/CT in assessment of response to chemotherapy and or radio therapy, follow-up and assessment of remissions and relapses in patients with lymphoma. In particular, a five-point scale (Deauville criteria) to grade response using PET-CT, which can be adapted to suit requirements for early-and late-therapeutic response assessment.

\section{Patients and Methods}

Study population: This retrospective study was conducted on Non Hodgkin lymphoma patients receiving chemotherapy presenting to Ain Shams University Hospitals Department of Radiology Unit of PET/CT since Jan. 2018-Apr. 2019; our study included 20 patients range of age between 21 and 68 years and a mean of 44 year. 12 out of the 20 patients $(60 \%)$ of all group study were male patients and 8 patients of all group study were female patients $(40 \%)$.

\section{Inclusion criteria:}

- Patients of any age group \& both sexes.

- Diagnosed with diffuse large B-cell type NonHodgkin lymphoma.

- Histologically confirmed.

- Receiving chemotherapy treatment.

\section{Exclusion criteria:}

Indolent type NHL and T cell type NHL.

Patients did not receive chemotherapy treatment.

Blood glucose level $>200 \mathrm{mg} / \mathrm{ml}$ at the time of examination that fails to be controlled. 
Any patient with absolute contraindication to expose to radiation e.g. pregnancy.

Ethical consideration: Administrative approval from Medical and Ethical Committee of Ain Shams University; informed consent will be obtained from the participants explaining study and method of examinations. Confidentiality of data obtained will be explained to research participations.

Study tools: The PET/CT at Ain Shams University Hospitals. Is a general electric discovery brand that will be used in this study.

\section{Patient preparation:}

1- Fasting 4-6 hours prior to procedure.

2- Sufficient hydration is a must to ensure low concentration of FDG in urine.

3- No strenuous exercises at least a day before imaging.

Procedure: Besides the review of, all patients subjected to:

1- After injection of FDG patient should remain seated and calm for $30-60 \mathrm{~min}$.

2- Patient should void immediately before imaging to reduce bladder activity.

3- The patient should lie with arms above head during imaging (20-45 min).

4- The glucose level should less than $200 \mathrm{mg} / \mathrm{dl}$ and above $70 \mathrm{mg} / \mathrm{dl}$ before imaging.

\section{Statistical analysis:}

Diagnostic performance parameters were calculated in the form of sensitivity, specificity, PPV, NPV and accuracy. The chi-square test was used to evaluate the statistical significance of the differences in sensitivity and specificity (a two-sided $p$ value $<0.05$ was considered significant) the collected data will be revised, coded, tabulated and introduced to a PC using Statistical Package for Social Science (SPSS 20.0.1 for Windows; SPSS Inc, Chicago, IL, 2001).

\section{Results}

This retrospective study consists of 20 patients selected from the archive of the Department of Radiology at Ain-Shams University, throughout the period Jan. 2018-Apr. 2019. In our study included 20 patients range of age between 21 and 68 years and a mean of 44 year. 12 out of the 20 patients $(60 \%)$ of all group study were male patients and 8 patients of all group study were female patients $(40 \%) 10 \%$ of the patients were stage I,
$20 \%$ as stage II, $30 \%$ stage III, $40 \%$ stage IV. And so, 14 patients $(70 \%)$ presented with advanced stages III and IV as in (Table 1).

Table (1): Patient characteristics at presentation are summarized in it.

\begin{tabular}{lcc}
\hline Characteristics & Patients $(\mathrm{n}=20)$ & Percent $(\%)$ \\
\hline Age (yrs.): & & \\
$\quad$ Range & $21-68$ & \\
Mean \pm SD & $44.58 \pm 13.46$ & \\
Gender: & & \\
Male & 12 & $60 \%$ \\
Female & 8 & $40 \%$ \\
Stage at presentation: & & \\
Stage I & 2 & $10 \%$ \\
Stage II & 4 & $20 \%$ \\
Stage III & 6 & $30 \%$ \\
Stage IV & 8 & $40 \%$ \\
\hline
\end{tabular}

Table (1): Showing patient's characteristics in all patients included in study.

The sites of the lymph nodes groups involved were classified as being either supra-diaphragmatic, infra-diaphragmatic or both (Table 2) \& Chart. 11 patients had supra-diaphragmatic lymph nodes involved, 2 patients had infra-diaphragmatic lymph nodes involved and 7 patients had both supra and infra-diaphragmatic involvement.

Table (2): Different sites of lymph nodes involvement in study group.

\begin{tabular}{lcc}
\hline $\begin{array}{l}\text { Sites of lymph } \\
\text { nodes groups }\end{array}$ & Number of patients & Percent $\%$ \\
\hline Supra and infra & 7 & $35 \%$ \\
Infra & 2 & $10 \%$ \\
Supra & 11 & $55 \%$ \\
\hline Total & 20 & 100 \\
\hline
\end{tabular}

More than $50 \%$ of our patients in the study had supra-diaphragmatic nodal involvement however, only $10 \%$ had infra diaphragmatic nodal involvement and 35\% had both supra and infra involvement.

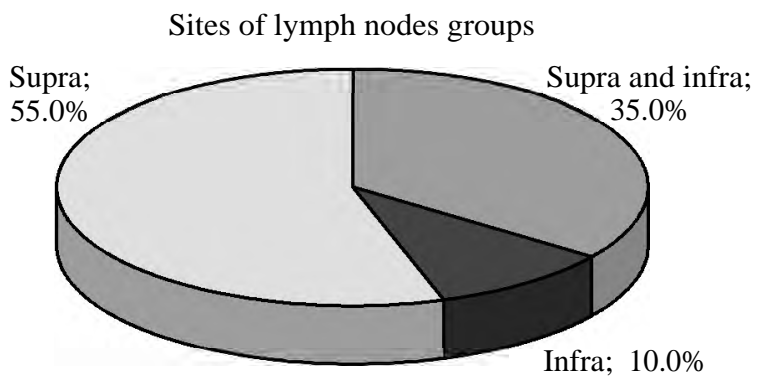

Pie Chart of Table (2): Showing different sites of nodal lesions in the study groups. 
In our study, 12 patients $(58.3 \%)$ had extranodal involvement and were as follow: 7 patients (41.7) had only one extranodal affection site and 5 patients $(45 \%)$ had more than one extranodal affection site.

Table (3): Extra-nodal affection in study group.

\begin{tabular}{lcc}
\hline Sites of lymphatic lesion & Number of patients & Percent $\%$ \\
\hline Nodal alone & 8 & $40 \%$ \\
Nodal and extra-nodal & 8 & $40 \%$ \\
Extra-nodal alone & 4 & $20 \%$ \\
\hline Total & 20 & 100 \\
\hline Sites of extranodal & Number of patients & Percent \\
involvement & $\mathrm{N}=12$ extranodal & $\%$ \\
\hline One extra-nodal site & 7 & $58.3 \%$ \\
More than one site & 5 & $41.7 \%$ \\
\hline
\end{tabular}

As shown in the following (Table 4) different extra-nodal site were expressed in our study with splenic involvement was the highest site with $25 \%$ followed by head and neck involvement with $20 \%$, pleura-pulmonary with $15 \%$, liver and other GIT involvement with $10 \%$ each, bone \& bone marrow with $10 \%$, genitor-urinary with $5 \%$ and skin \& soft tissue with also $5 \%$.

Table (4): The different sites of the extra-nodal disease in study groups.

\begin{tabular}{lcc}
\hline $\begin{array}{l}\text { Sites of extranodal } \\
\text { involvement }\end{array}$ & Number of patients & Percent (\%) \\
\hline Spleen & 5 & $(25 \%)$ \\
Liver & 2 & $(10 \%)$ \\
Pleuro-pulmonary & 3 & $(15 \%)$ \\
Other GIT & 2 & $(10 \%)$ \\
Bone \& bone marrow & 2 & $(10 \%)$ \\
Genitor-urinary & 1 & $(5 \%)$ \\
Head \& neck & 4 & $(20 \%)$ \\
Skin \& soft tissue & 1 & $(5 \%)$ \\
CNS & 0 & $(0 \%)$ \\
\hline
\end{tabular}

After end of treatment, by CT 9 patients had a CR, 7 patients had PR, one patient had a SD and 3 patients had PD. In comparison, by the basis of $\mathrm{PET} / \mathrm{CT}$ and Deauville criteria, 12 patients had a CR (Deauville 1, 2 and 3), 6 had a PR, no patient had SD and 2 patients had PD. As shown in the following (Table 5).
Table (5): Showing different response assessment percent of patients at the end of treatment by $\mathrm{CT}$ and PET/CT.

\begin{tabular}{|c|c|c|c|c|c|c|c|c|}
\hline \multirow{3}{*}{$\begin{array}{l}\text { Post- } \\
\text { treatment } \\
\text { CT }\end{array}$} & \multicolumn{8}{|c|}{ Post-treatment PET CT } \\
\hline & \multicolumn{2}{|c|}{$\mathrm{CR}$} & \multicolumn{2}{|c|}{ PR } & \multicolumn{2}{|c|}{ PD } & \multicolumn{2}{|c|}{ Total } \\
\hline & $\mathrm{N}$ & $\%$ & $\mathbf{N}$ & $\%$ & $\mathrm{~N}$ & $\%$ & $\mathrm{~N}$ & $\%$ \\
\hline $\mathrm{CR}$ & 9 & 45 & 0 & 0 & 0 & 0 & 9 & 45 \\
\hline PR & 2 & 10 & 5 & 25 & 0 & 0 & 10 & 35 \\
\hline SD & 0 & 0 & 0 & 0 & 1 & 5 & 1 & 5 \\
\hline PD & 1 & 5 & 1 & 10 & 1 & 5 & 3 & 15 \\
\hline Total & 12 & 60 & 6 & 30 & 2 & 10 & 20 & 100 \\
\hline
\end{tabular}

Table (6): Showing different response assessment by CT and $\mathrm{PET} / \mathrm{CT}$ and their chi-square and $p$-value denoting that there are significant concordance between CT and PET/CT in assessment of response to treatment $p$-value was significant $<0.05$.

\begin{tabular}{|c|c|c|c|c|c|c|}
\hline \multirow{2}{*}{$\begin{array}{l}\text { Response } \\
\text { to } \\
\text { treatment }\end{array}$} & \multicolumn{2}{|c|}{$\begin{array}{l}\text { Post-treatment } \\
\text { CT }\end{array}$} & \multicolumn{2}{|c|}{$\begin{array}{l}\text { Post-treatment } \\
\text { PET CT }\end{array}$} & \multicolumn{2}{|c|}{$\begin{array}{l}\text { Chi- } \\
\text { Square }\end{array}$} \\
\hline & $\mathrm{N}$ & $\%$ & $\mathrm{~N}$ & $\%$ & $x^{2}$ & $p$-value \\
\hline $\mathrm{CR}$ & 9 & 45 & 12 & 60 & 0.401 & 0.527 \\
\hline PR & 7 & 35 & 6 & 30 & 0.005 & 0.946 \\
\hline SD & 1 & 5 & 0 & 0 & 0.034 & 0.853 \\
\hline PD & 3 & 15 & 2 & 5 & 0.278 & 0.598 \\
\hline Total & 20 & 100 & 20 & 100 & 0.000 & 1.000 \\
\hline
\end{tabular}

$p$-value *(significant) when being $<0.05$.

Table (7): Represents comparison in response assessment between $\mathrm{CT}$ and PET CT showing high PET-CT sensitivity $100 \%$ with significant specificity.

\begin{tabular}{|c|c|c|c|c|c|c|}
\hline \multirow{3}{*}{$\begin{array}{l}\text { Post- } \\
\text { treatment } \\
\text { CT }\end{array}$} & \multicolumn{6}{|c|}{ Post-treatment PET CT } \\
\hline & \multicolumn{2}{|c|}{ Positive } & \multicolumn{2}{|c|}{ Negative } & \multicolumn{2}{|c|}{ Total } \\
\hline & $\mathrm{N}$ & $\%$ & $\mathrm{~N}$ & $0 \%$ & $\mathrm{~N}$ & $\%$ \\
\hline Positive & 8 & 40 & 3 & 15 & 11 & 55 \\
\hline Negative & 0 & 0 & 9 & 45 & 9 & 45 \\
\hline Total & 8 & 40 & 12 & 60 & 20 & 100 \\
\hline
\end{tabular}

The following: Table (8) and Chart showing that PET/CT had high sensitivity in assessment of complete remission (negative finding) after the end of treatment also PET/CT had high sensitivity in detection of active residual disease (positive finding).

Table (8): Showing that PET/CT had high sensitivity and specificity in assessment of therapeutic response.

\begin{tabular}{lcccc}
\hline Sens. & Spec. & + PV & $-P V$ & Accuracy \\
\hline $100.0 \%$ & $75.0 \%$ & $72.7 \%$ & $100.0 \%$ & $85.0 \%$ \\
\hline
\end{tabular}



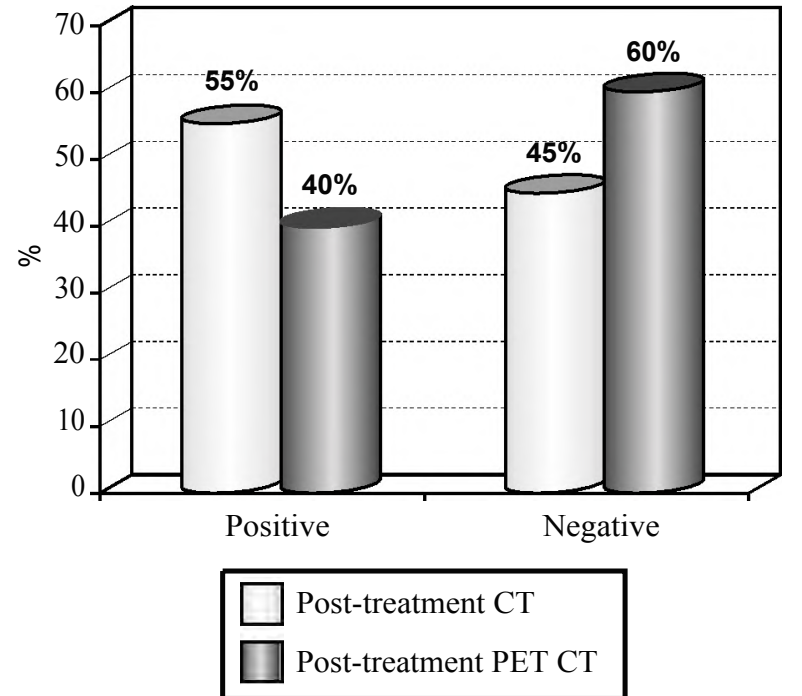

Chart of Table (8): Showing that PET/CT had high sensitivity in assessment of complete remission (negative finding) and active residual disease (positive finding).

\section{Case presentation:}

Male 57 years old presented by neck swelling and abdominal pain and mass Biopsy revealed non Hodgkin lymphoma Diffuse Large B Cell Lymphoma (DLBCL) patient received interim 8 cycles of chemotherapy; post-treatment finding Partial Response (PR), Deauville 4 (FDG uptake moderately More than liver) CT \& PET/CT show same result as illustrated in Figs. $(1,2)$.

Female patient age 63 years presented by B symptoms (fever, weight loss, night sweating) and left upper abdominal pain. Initial CT reveal moderate splenomegaly and splenic focal lesion measured about 4 X $3 \mathrm{~cm}$ Fig. (3A). Post-treatment finding complete remission, Deauville 3 (FDG uptake less than the liver) CT discordance with $\mathrm{PET} / \mathrm{CT}$ finding this property of PET/CT differentiate fibrotic and necrotic tissue from residual mass Figs. $(3 \mathrm{~B}, 4)$.

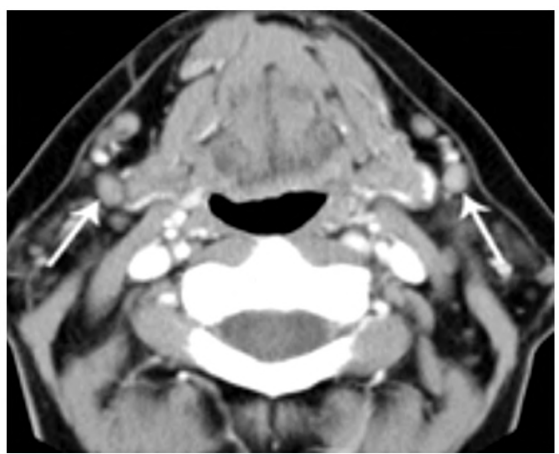

(A)

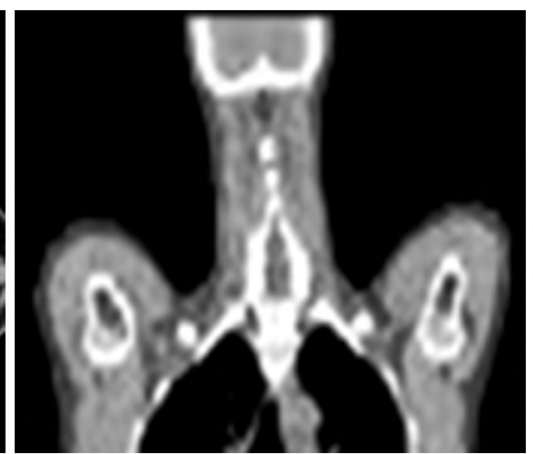

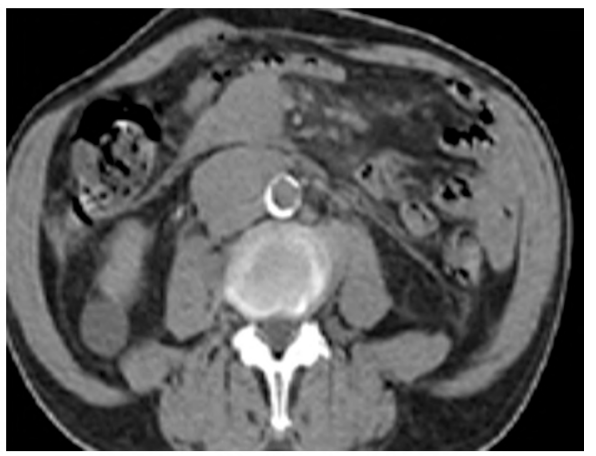

(B)

Fig. (1): Post-treatment CT (A) Corona $1 \&$ axial CT image of neck showing no pathologically enlarged cervical lymph nodes (B) Axial CT image of the abdomen showing enlarged para-aortic lymph nodes.

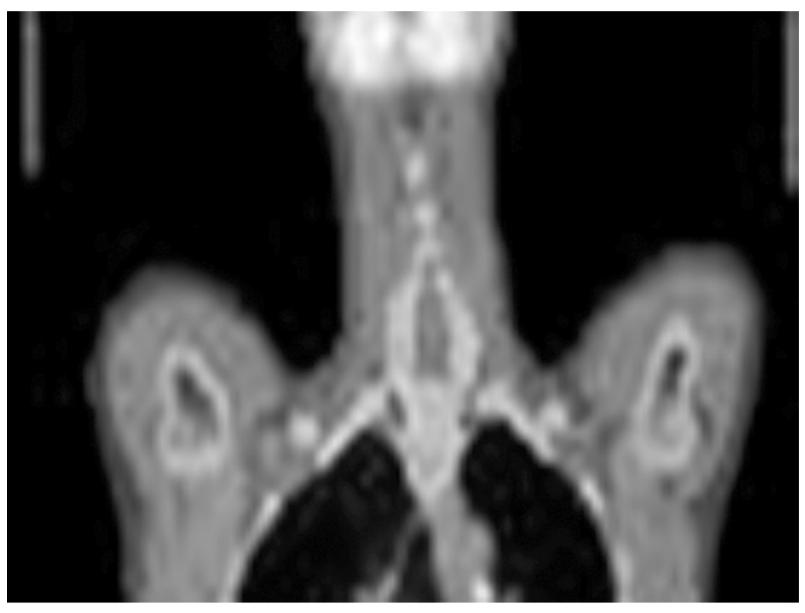

(A)

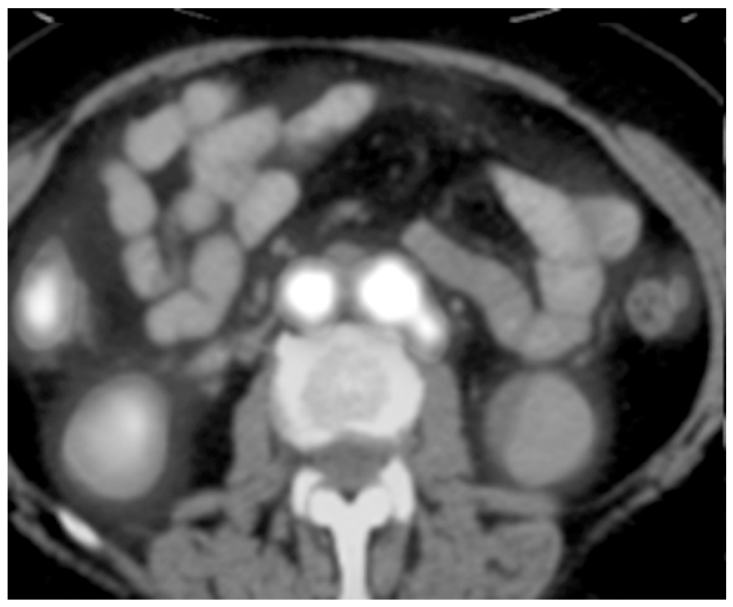

(B)

Fig. (2): Post-treatment PET/CT (A) Coronal PET/CT images of neck showing no FDG uptake cervical lymph nodes. (B) Axial PET/CT image of the abdomen showing FDG avid uptake of the para-aortic lymph nodes. 

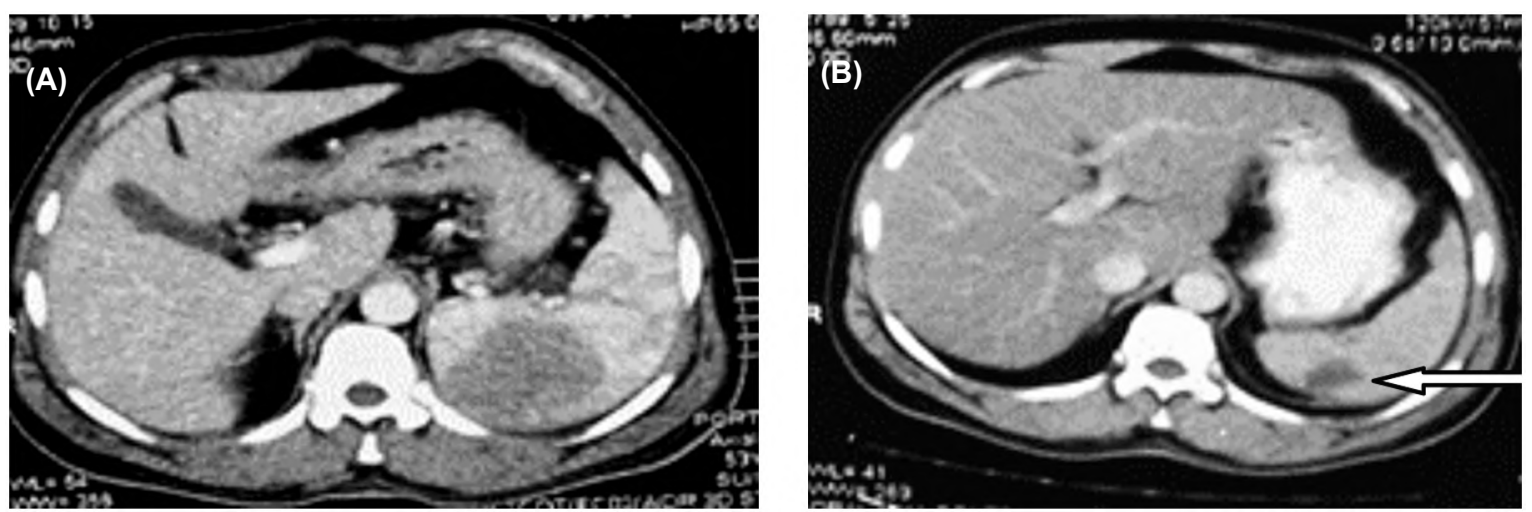

Fig. (3): (A) Axial CT image of the abdomen showing posterior pole hypodense splenic focal lesion measured about $(4 \mathrm{X} 3 \mathrm{~cm}$ (B) Post-treatment AXIAL CT of abdomen showing posterior pole hypodense splenic focal lesion measured about 2 X $1.5 \mathrm{~cm})$.
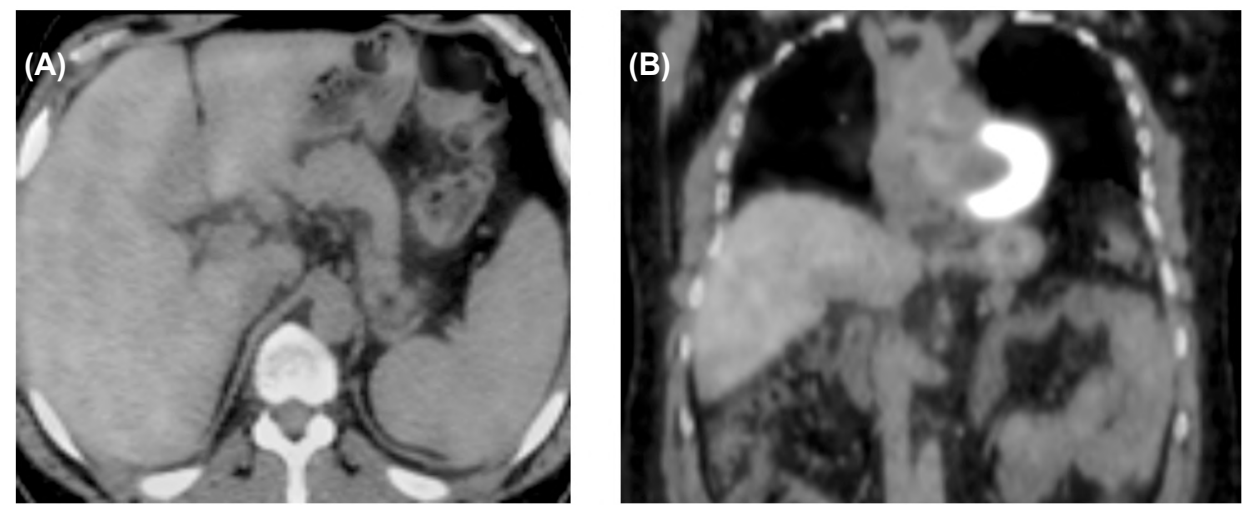

Fig. (4): (A, B) Axial \& coronal PET/CT image showing no FDG avid uptake and residual splenic focal lesion was inactive.

\section{Discussion}

Lymphoid neoplasms are broadly divided into Hodgkin Disease (HD) and Non-Hodgkin Lymphoma (NHL). Hodgkin lymphoma accounts for less than $1 \%$ of all cases of cancer, Non-Hodgkin Lymphoma (NHL) accounts for about $4-5 \%$ of all cases of cancer [9].

Aggressive types of NHL constitute the most short survival but chemo-sensitive lymphomas and high curability chance with combination chemotherapy; Diffuse Large B Cell lymphoma (DLBC) as one of the aggressive types of NHL, constituting the commonest of the hematological malignancies as a whole and accounting for around $48 \%$ of all non-Hodgkin lymphomas [10]

NHL diffuse large B cell characterized by high nodal and extra nodal involvement and usually presented in advanced stages (stage III and IV), but gain the same chance of being cured with combination immune-chemotherapy [11]

In this study, both the CT and FET scans were done during shallow breathing and instructed the patients not to move during the study to minimize the mis-registration artifacts in agreement with Townsend 2004 [12].

We ensured keeping the patients in a relatively warm environment in order to decrease the brown fat uptake which was very effective in evaluation of the supradiaphragmatic lymph nodes. The brown fat uptake is one of the causes of false positive results in concordance with Tatsumi 2005 [13]

The advantage of metabolic imaging is its ability to distinguish viable metabolically active tissue from scars, potential to detect functional changes in response to chemo or radio-therapy before there is any change in clinical or radiological size of a mass and detection is dependent on the intensity of the activity rather than the lesion size [14]

SUV is a simplified index of $18 \mathrm{~F}-\mathrm{FDG}$ uptake and provides a relative indication of the degree of metabolism within the lesion being evaluated. It is a unit that can be understood as the concentration of $18 \mathrm{~F}-\mathrm{FDG}$ with in a lesion divided by the concentration of radiotracer distributed throughout the body [9]. 
Our study included 20 patients range of age between 21 and 68 years and a mean of 44 year. 12 out of the 20 patients (60\%) of all group study were male patients and 8 patients of all group study were female patients $(40 \%)$. Keeping with the results of [15].

In their study that nodal alone involvement 8 (40\%) nodal and extra-nodal $8(40 \%) 4$ patients (20\%) presented with only one extranodal primary site which resemble our data that revealed $20 \%$ of total patients had only one primary extra-nodal site [16].

Also for extra-nodal involvement different organ reported was: 5 patients had splenic involvement, 4 patients had head and neck involvement, 3 patients showed pulmonary nodules 2 patients had hepatic involvement, 2 patients had other GIT involvement, 2 patient with osseous involvement and 1 patients showed soft tissue \& skin affection, and one patient had genitor-urinary affection, no patient with CNS involvement. Splenic and GIT involvement was the most common extra nodal site keeping with results of [17].

Both CT and PET/CT have demonstrated high sensitivity for extra-nodal lesions, but PET/CT may be particularly helpful in discriminating benign from malignant lesions that was in concordance with [18]

One of the most challenging aspects in the imaging of lymphomas is the assessment of response to treatment. Differentiation of active lesions represents a problem of interpretation for nonHodgkin's lymphoma. Thus accurate staging is the basis for the selection of an appropriate therapeutic approach, in order prevent over or under treatment as well as to minimize morbidity related to the radio-chemotherapy regimens given [7]. The CT portion of PET/CT exams plays a crucial role in the response evaluation of lymphoma patients. According to the IHP, for response evaluation of lymphoma patients which was applied in this study, if there is residual metabolic activity associated with morphological reduction, the response is either Partial Response (PR) or Stable Disease (SD) depending on how much reduction in size is reported by $\mathrm{CT}$, i.e. if the reduction in size was by $>50 \%$, it is considered PR while if the reduction was by $<50 \%$, it is considered SD [19].

Visual assessment using (Deauville criteriafive-point scale) were suited to assess different degrees of response after end of treatment, and has been developed to score images. Deauville criteria classified into scores that represent lesions activity with score 1 showing no uptake above background, score 2 showing uptake at an initial site that is less than or equal to mediastinum, score 3 showing uptake at an initial site that is greater than mediastinum but less than or equal to liver, score 4 showing uptake at an initial site that is moderately increased compared to the liver at any site and score 5 showing uptake at an initial site that is markedly increased compared to the liver [20]

On interpretation of PET/CT after end treatment; on the basis of the IHP, 9 patients had a CR, 3 had PD, 7 had a PR and one patient had SD. In comparison, by the basis of Deauville criteria, 12 patients had a CR, 2 had PD, 6 had a PR and no patient had SD. Our study revealed high sensitivity and specificity of PET/CT over CT alone and this in concordance with some trials [21].

Also we revealed that end-of-treatment PET/CT is better for remission assessment than CT and this is matched with the results of Barrington SF 1 \& Mikhaeel NG. [22].

\section{Limitation:}

- Pathological confirmation was not available for most of the residual lesions, for which diagnosis was based on imaging criteria, clinical/laboratory data and follow-up.

- Small sample size of post-treatment patients so our results in this group need to be further evaluated on a larger sample size.

- The proposed five-point scale is a visual measure of FDG PET/CT response assessment; the risk of a degree of subjectivity in the interpretation of FDG avidity has not been eliminated.

\section{Conclusion:}

$\mathrm{PET} / \mathrm{CT}$ is the technique of choice for patients with Non Hodgkin lymphoma as it has been demonstrated that 18F-FDG PET/CT is extremely useful tool for therapy response assessment especially for those patients with residual disease. $\mathrm{PET} / \mathrm{CT}$ as a tool of assessment of response can discriminates between active lesion and non-active necrotic or fibrotic lesion, response assessment according to the Deauville criteria classification represent a practical framework used for interim PET CT follow-up of non-Hodgkin lymphoma. Lymphoid neoplasms are broadly divided into Hodgkin Disease (HD) and Non-Hodgkin's Lymphoma (NHL). Non-Hodgkin lymphoma accounts for about $5 \%$ of all cases of cancer with greater predilection to disseminate to extra-nodal sites aggressive NHL usually presented by nodal and or extra-nodal involvement and although it is an 
aggressive lymphoma, many cases show complete remission denoting that it is a chemo-sensitive tumor.

In recent years, imaging with Positron Emission Tomography (PET) for tumor staging and therapy control has been introduced. Rather than anatomic information, it provides physiologic information on glucose uptake and metabolism. The main drawback of PET in tumor imaging is the virtually complete absence of anatomic landmarks, which impedes precise localization of lesions combined PET/CT facilitates the separation of normal physiologic uptake from pathologic uptake, provides accurate localization of functional abnormalities, and reduces the incidence of false-positive and false-negative imaging studies. The imaging time for a whole-body scan is also markedly reduced, enhancing patient comfort and convenience.

In response to developments involving PET$\mathrm{CT}$, recommendations from the ICML (International Conference on Malignant Lymphoma) imaging group have been made to update practice. These include guidance on reporting of PET-CT for staging and response assessment using the Deauville criteria. Although Deauville criteria has specific qualitative criteria for response based on a visual activity of the lesion, collection of additional data as SUV is recommended so as to develop a database suitable for additional information to refine the response metrics for a given tumor and therapy and as potential prognosticators. The assessment of therapeutic response in our study by using IHP (international harmonizing project). And correlated with visual assessment using (Deauville criteriafive-point scale). The result of this 20 patients study revealed discordance between contrast enhanced CT \& PET/CT finding, PET/CT had high sensitivity $(100 \%)$ and specificity $(75 \%)$ for the evaluation of therapeutic response than CT according to the Deauville criteria.

\section{References}

1- PÁEZ J.M. NOGUEIRAS ALONSO and A. SERENA PUIG: 18 F-FDG-PET/CT in lymphoma: Two decades of experience, Rev. Esp. Med. Nucl. Imagen. Mol., 31 (6): 340-9, 2012.

2- DRAKE M., KETTLE P., LYNCH T., MORRIS C., PAGE W., SHIELS A. and VENKATRAMEN L.: The Role of PET/CT in Lymphoma Part I, PET/CT in Clinical Practice, 1 (4): 16-9, 2007.

3- DESSAIN S.K., SPEARS J.L. and ARGIRIS A.: Hodgkin Disease. www.emedicine.com Updated, 2015.

4- FABIO M. PAES, DIMITRIOS G. KALKANIS, PANAGIOTIS A. SIDERAS and ALDO N. SERAFINI: FDG $\mathrm{PET} / \mathrm{CT}$ of extranodal involvement in non-Hodgkin Lym- phoma and Hodgkin Disease, Radio Graphics, 30: 26991, 2010.

5- ANSELL STEPHEN M. and JAMES O. ARMITAGE: Positron Emission Tomographic Scans in Lymphoma: Convention and Controversy, Mayo Clin. Proc., 87 (6): 571-80, 2012.

6- GALLAMINI A., BIGGI A., CHAUVIE S., et al.: International Validation Study for Interim PET in ABVDTreated, Advanced-Stage Hodgkin Lymphoma: Interpretation Criteria and Concordance Rate among Reviewers, the Journal of Nuclear Medicine, 54: 683-90, 2013.

7- BARRINGTON F., MIKHAEEL N., KOSTAKOGLU L., et al.: Role of Imaging in the Staging and Response Assessment of Lymphoma: Consensus of the International Conference on Malignant Lymphomas Imaging Working Group, Journal of Clinical Oncology, 32: 3048-58, 2014.

8- CHESON B.D. and KOSTAKOGLU L.: FDG-PET for Early Response Assessment in Lymphomas: Part 2-Diffuse Large B-Cell Lymphoma, Use of Quantitative PET Evaluation. Oncology (Williston Park), 31 (1): 71-6, 2017.

9- WORKMAN E.R. and COLEMAN E.R.: PET/CT Essentials for Clinical Practice Springer Science, Business Media, LLC: 3-12, 2006.

10- SMITH A., CROUCH S., HOWELL D., et al.: Impact of age and socioeconomic status on treatment and survival from aggressive lymphoma: A UK population-based study of diffuse large B-cell lymphoma. Cancer Epidemiol., 39 (6): 1103-12, 2015.

1 1- ZIEPERT M., HASENCLEVER D. and KUHNT E.: Standard International prognostic index remains a valid predictor of outcome for patients with aggressive CD20+ B-cell lymphoma in the rituximab era. J. Clin. Oncol., 28 (14): 2373-80, 2010.

12- TOWNSEND D.W., CARNEY J., YAP F., et al.: PET/CT Today and Tomorrow. J. Nucl. Med., 45: 4S-14S, 2004.

13- TATSUMI M., COHADE C., NAKAMOTO Y., et al.: Direct comparison of FDG PET and CT findings in patients with lymphoma: Initial experience. Radiology, 237: 103845, 2005.

14-ANTOCH G., KUEHL H., KANJA J., et al.: Dual-modality $\mathrm{PET} / \mathrm{CT}$ scanning with negative oral contrast agent to avoid artifacts: Introduction and evaluation. Radiology, 230: 879-85, 2004.

15-LEHNERS N., KRÄMER I., SAADATI M., et al.: Analysis of prognostic factors in patients with newly diagnosed diffuse large B-cell lymphoma and skeletal involvement. BMC Cancer, 17 (1): 128, 2017.

16- CASTILLO J.J., WINER E.S. and OLSZEWSKI A.J.: Sites of extranodal involvement are prognostic in patients with diffuse large B-cell lymphoma in the rituximab era: An analysis of the Surveillance, Epidemiology and End Results database. Am. J. Hematol., 89 (3): 3 10-4, 2014.

17- SHAHID R., GULZAR R., AVESI L., et al.: Immunohistochemical Profile of Hodgkin and Non-Hodgkin Lymphoma. J. Coll. Physicians. Surg. Pak., 26 (2): 103-7, 2016.

18- BIGGI A., GALLAMINI A., CHAUVIE S., et al.: International Validation Study for Interim PET in ABVDTreated, Advanced-Stage Hodgkin Lymphoma: Interpre- 
tation Criteria and Concordance Rate among Reviewers, the Journal of Nuclear Medicine, 54: 683-90, 2013.

19- CHESON B., PFISTNER B., JUWEID M., et al.: Revised Response Criteria for Malignant Lymphoma. Journal of Clinical Oncology, Vol. 25, No. 5, 579-86, 2007.

20- AWAN U.E., SIDDIQUI N., SAADULLAH M., et al.: FDG-PET scan in assessing lymphomas and the application of Deauville Criteria. J. Pak. Med. Assoc., 63 (6): 725 30, 2013.
21- METSER U., MOHAN R., BECKLEY V., et al.: FDG PET/CT Response Assessment Criteria for Patients with Hodgkin's and Non-Hodgkin's Lymphoma at End of Therapy: A Multiparametric Approach. Nucl. Med. Mol. Imaging, 50 (1): 46-53, 2016.

22- BARRINGTON S.F. and MIKHAEEL N.G.: PET Scans for Staging and Restaging in Diffuse Large B-Cell and Follicular Lymphomas. Curr. Hematol. Malig. Rep., 11 (3): 185-95, 2016.

\section{دور التصوير الطبقى بالإنبعاث البوزتروثى المدمج مع الآثعة المقطعية (PET/CT) فى تقيم الإستجابة العلاجية لسرطان الغلد البهاد اللمفاوية (الغير هودجكين)}

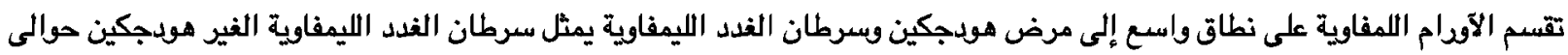

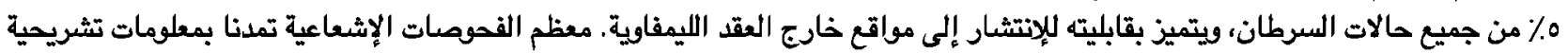

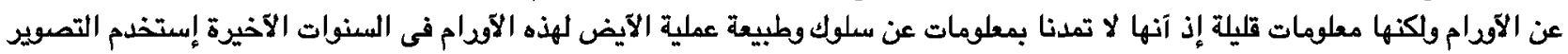

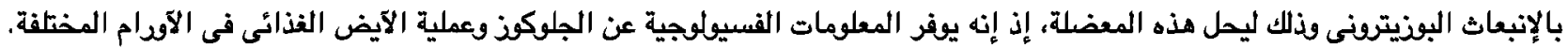

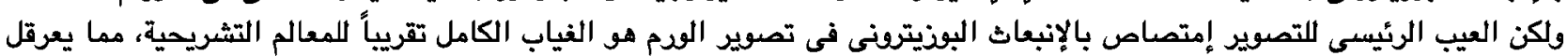

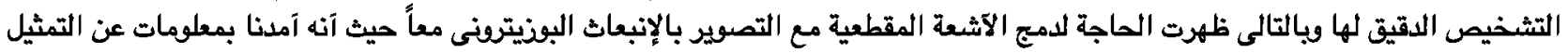

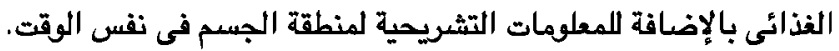

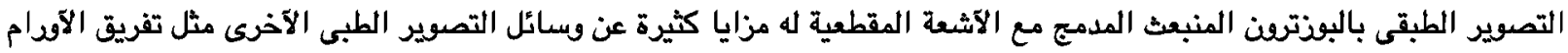

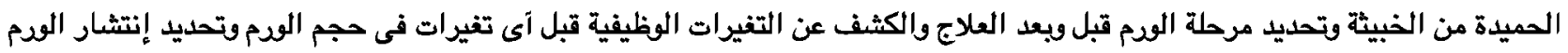

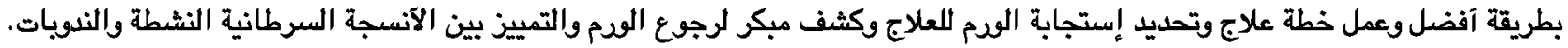

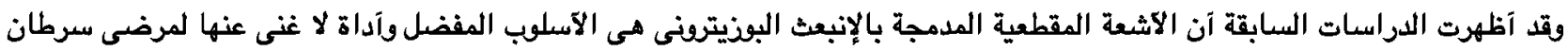

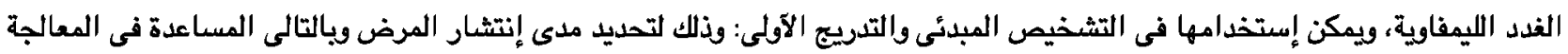

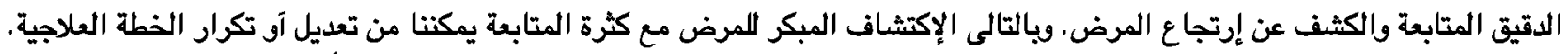

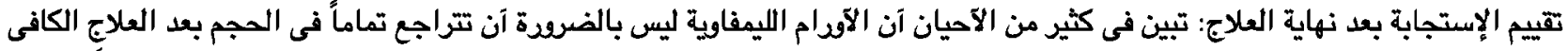

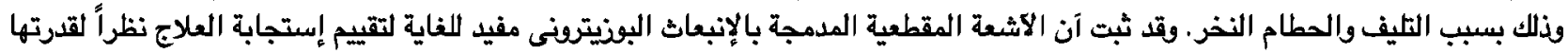

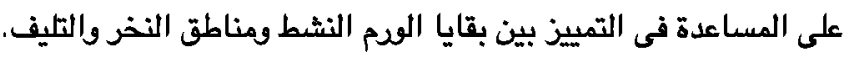

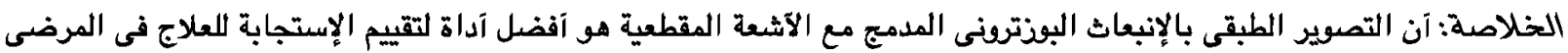

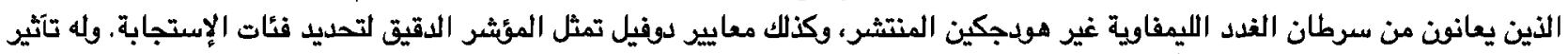

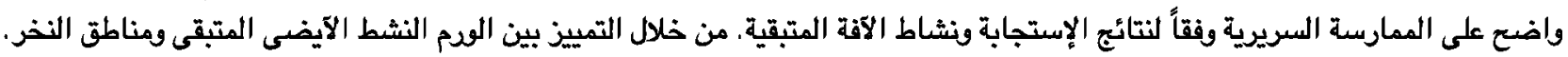

